\title{
Deux semaines en Roumanie avec Yorgos Théotokas
}

Two weeks in Romania with George Theotokas

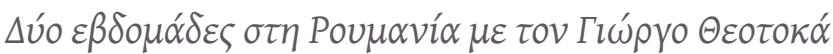

\section{Georges Kostakiotis}

\section{OpenEdition \\ Journals}

Édition électronique

URL : https://journals.openedition.org/ceb/9998

DOI : $10.4000 /$ ceb.9998

ISSN : 2261-4184

Éditeur

INALCO

Édition imprimée

ISBN : 978-2-85831-239-9

ISSN : 0290-7402

Référence électronique

Georges Kostakiotis, "Deux semaines en Roumanie avec Yorgos Théotokas », Cahiers balkaniques [En ligne], 44 | 2016, mis en ligne le 08 décembre 2017, consulté le 07 juillet 2021. URL : http:// journals.openedition.org/ceb/9998; DOI : https://doi.org/10.4000/ceb.9998

Ce document a été généré automatiquement le 7 juillet 2021.

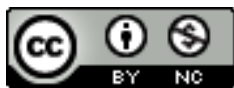

Cahiers balkaniques est mis à disposition selon les termes de la Licence Creative Commons Attribution - Pas d'Utilisation Commerciale 4.0 International. 


\title{
Deux semaines en Roumanie avec Yorgos Théotokas
}

\author{
Two weeks in Romania with George Theotokas

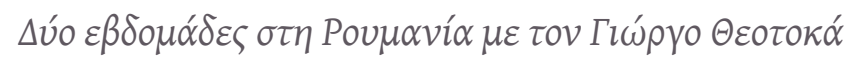

\section{Georges Kostakiotis}

1 En 1962, à l'occasion de la commémoration en Roumanie du cinquantième anniversaire de la mort de l'écrivain-dramaturge Ion Luca Caragiale, les écrivains Yorgos Théotokas et Spyros Melas ainsi que le poète Yannis Ritsos ont été invités à Bucarest pour représenter la Grèce. Théotokas a séjourné en Roumanie pendant deux semaines et toutes les informations que nous tenons de ce voyage proviennent de quatre articles

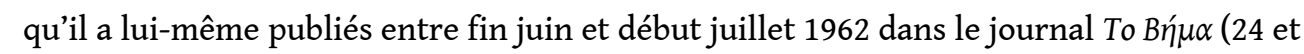
26 juin, ${ }^{\text {er }}$ et 4 juillet) avec lequel il collaborait depuis 1957.

2 Yorgos Théotokas - qui a été l'un des représentants les plus actifs de la littérature des années 1930 - continue alors à être très présent non seulement par sa production littéraire ${ }^{1}$, mais aussi en tant que critique littéraire et journaliste jusqu'à la fin de sa vie en 1966. Depuis 1961, il est également président du Théâtre National de Thessalonique, alors qu'en 1964-1965 il assurera à nouveau la direction de celui d'Athènes dont il a déjà été directeur en 1945-1946.

3 Théotokas a été accusé de flirter avec les idées fascistes et totalitaires ; aujourd'hui encore la critique littéraire a du mal à accepter et à justifier le silence de cette bourgeoisie intellectuelle de l'entre-deux-guerres, dont Théotokas reste un acteur majeur, devant la montée des extrémismes en Europe ${ }^{2}$. En effet, fervent partisan d'une Europe unie - longtemps avant la création du Marché commun -, il a cru voir dans les mouvements totalitaires un moyen d'arriver à cette union. En même temps, il n'a jamais considéré le fascisme ou le nazisme comme une menace qui pourrait toucher directement la Grèce. Au contraire, pour lui, le vrai danger, venant de l'intérieur même du pays, était le communisme.

4 Notons néanmoins qu'après la déclaration de guerre par l'Italie, il s'engage comme volontaire. Et il n'hésitera pas à condamner ouvertement la politique de 
Ioannis Metaxás ainsi que les nationalistes extrémistes ${ }^{3}$. Ajoutons que depuis 1954 il s'engage aux côtés de Georges Papandréou qu'il connaît depuis 1943 et, en 1956, il est candidat à Chios avec l'Union Démocratique de Sofoklis Venizélos contre l'Union radicale nationale (ERE) de Constantin Caramanlis.

5 Théotokas n'était pas un journaliste de métier, mais dans ses articles il a toujours été très sincère sans jamais hésiter à formuler des propos et des points de vue quitte à $\mathrm{y}$ revenir plus tard pour accepter de s'être trompé. Nous pouvons rappeler le cas de Constantin Cavafy ou, pour notre travail, concernant le voyage en Roumanie, le cas de Spyros Melas ${ }^{4}$. Celui-ci, invité lui aussi en Roumanie, avait été désavoué publiquement par Théotokas quand il avait exprimé ses sympathies pour l'occupant nazi.

Ce séjour en Roumanie est très profitable à Théotokas pour deux raisons. D'une part, c'est la première fois qu'il a l'occasion de voyager dans un pays communiste. Le pays des Carpates sera son premier contact avec ce monde et, pour un homme qui a combattu le communisme avec acharnement, nous pouvons dire que ce voyage restera une étape importante de son parcours intellectuel. En décembre de la même année 1962, il aura l'occasion de visiter l'Union soviétique.

7 D'autre part, la Roumanie devient pour Théotokas le lieu de rencontre avec le monde balkanique; outre les contacts qu'il a eus avec des intellectuels roumains, l'écrivain insiste sur ses échanges avec un poète serbe et un écrivain bulgare.

Nous essaierons ici d'esquisser ces deux aspects afin de comprendre d'une part l'évolution de sa pensée politique et d'autre part le rôle qu'il accorde à la Grèce dans le monde des Balkans.

\section{Le premier voyage en pays communiste}

9 C'est une expérience inouïe pour Yorgos Théotokas que de se trouver dans un pays au-delà du rideau de fer; il regarde et observe ce monde sans que rien ne lui échappe. Ses commentaires et les impressions de son premier contact avec la capitale roumaine, l'observation des éditions littéraires et des ventes en librairies, la fréquentation des églises ou même des restaurants montrent son très vif intérêt pour le monde qui se dévoile à lui.

10 La beauté de la ville, l'espace, les jardins, les lacs et les grandes avenues, mais aussi la propreté, donnent une image d'une ville agréable à vivre ; fatalement, il la comparera à Athènes, pour noter que la capitale grecque a depuis longtemps perdu des plaisirs innocents tels que trouver facilement une place pour sa voiture ou flâner à pied dans des rues peu encombrées par la circulation.

11 Concernant les commerces, il souligne qu'il y en a beaucoup, de petits et de grands, avec une abondance d'articles pour la maison et l'habillement même si la qualité reste médiocre. Il souligne que les magasins sont très fréquentés; il utilisera à deux reprises le terme de «foule» pour nous donner une image opposée à celle que le monde occidental a toujours véhiculée à propos du monde communiste.

12 En effet, l'écrivain semble agréablement surpris par un univers qu'il attendait différent, plus distant et certainement plus attaché à la politique ou à l'idéologie politique. Or il est en contact avec des gens chaleureux, qui investissent leur ville et profitent pleinement de leur quotidien, complètement détachés de la politique. Il note que les gens sont absorbés et préoccupés par leur travail, les courses et leur vie privée. 
Plus tard, lors de sa visite en Russie, il notera avec beaucoup d'insistance :

Ne me demandez pas ce à quoi les gens pensent, ils pensent à leur femme ou à leur petite amie. Ils pensent à leurs enfants, à leur maison, aux questions de santé, à leur travail, à leur carrière. Ils pensent aux livres qu'ils lisent, parce qu'ils lisent beaucoup. En un mot, ils pensent à leur vie. Croire que les gens en Union soviétique ou ailleurs ne font que penser à la politique est enfantin ${ }^{5}$.

Néanmoins, il note que la vie ne lui semble pas insouciante pour tous : dans quelques restaurants, on rencontre de petits orchestres et des couples sages dansent; les gens rentrent tôt chez eux. Quant à l'alimentation, ces gens donnent l'impression continue-t-il -, qu'ils se nourrissent bien, les enfants sont soignés et ils ont les couleurs de la santé. Avec ces remarques quelque peu naïves, nous pouvons facilement en déduire que Théotokas est arrivé en Roumanie avec les clichés de la propagande anticommuniste selon laquelle les gens seraient mal nourris, mal habillés et en mauvaise santé dans des villes aux rues désertes et aux commerces vides de clients et de marchandises. Dans ce même esprit, il note que « dire que ces gens ont faim et qu'ils sont sur le point de se révolter contre le système, c'est naif et il ne faut pas le répéter ${ }^{6} »$. Ainsi, l'écrivain accepte sans difficulté que le régime communiste en Roumanie ait pu trouver son équilibre et dépasser les premières très grandes difficultés auxquelles il a eu à faire face. Autrement dit, la Roumanie, qu'il découvre, dément les stéréotypes de l'imaginaire occidental.

Effectivement, la Roumanie de 1960 traverse une période de relative prospérité 7 L'écrivain semble impressionné par les travaux de constructions menés sur un rythme rapide, non seulement à Bucarest, mais aussi à Ploiesti et à Constantza où de vieux quartiers sont reconstruits avec des immeubles populaires énormes, volumineux et uniformes aux couleurs claires dans l'esprit du régime qui vise à la collectivisation de la société et à l'industrialisation rapide de l'État, note-t-il.

Ces constructions intéressent particulièrement Yorgos Théotokas; elles incarnent à ses yeux la politique du pays dans sa volonté de résoudre le problème du logement pour toute la population en offrant aux ouvriers des appartements à loyers bas. L'écrivain s'informe sur les différents types d'habitation, leur capacité ainsi que les diverses facilités proposées. D'ailleurs, il nous rapporte toutes ces informations très précises en notant chaque fois "comme il m'a été dit»; et il précise que ce vaste programme devrait être accompli en 1975. Durant une excursion organisée, il a eu l'occasion de voir de près ce type de constructions et d'y séjourner, dans la célèbre station balnéaire de Mamaia à proximité de Constantza. Théotokas ne cache pas son admiration pour l'intérêt que l'État manifeste pour le repos des ouvriers et il parle sans réserve « d'un progrès social qu'il ne peut pas nier ${ }^{8}$ "; impressionné par la taille des constructions, il les compare au Hilton d'Athènes, en construction depuis 1959, qui sera inauguré le 20 avril 1963.

17 Ce regard admiratif sera quelque peu freiné : l'écrivain ne manquera pas d'émettre quelques réserves face au gigantisme de cet ouvrage qui a comme but la collectivisation; il parle d'une " vie massive ${ }^{9}$ » où l'individu semble écrasé.

Dans ce même esprit, une autre visite organisée durant son séjour, dans la vallée de la Prahova cette fois-ci, lui donnera l'occasion d'arriver jusqu'à Brasov, de visiter un domaine agricole et de recueillir des informations sur l'organisation des exploitations. Durant cette visite aussi, Théotokas paraît convaincu des bonnes intentions du système communiste, il semble apprécier la mise en place d'un univers juste où chaque 
agriculteur possède sa maison ainsi qu'un terrain pour ses cultures personnelles qu'il peut même vendre au marché - comme en Grèce, insiste-t-il. Quant à la rémunération, il trouve juste que chaque ouvrier soit payé selon les journées de travail effectuées. En revanche, les réserves de l'écrivain sont toujours vives sur l'ampleur de cette présence de l'État où tout est public, jusqu'à la moindre petite buvette ou la dernière péniche qui flotte sur le Danube.

La question qui semble préoccuper Théotokas après avoir constaté que ce système propose une organisation plus juste de la société est la place que la personne, en tant qu'individu, occupe dans ce monde, dans ce type d'organisation sociale. Pour lui qui croit - comme il le dit lui-même - en la personne humaine, à son originalité et à sa liberté, une telle " collectivisation ${ }^{10}$ » lui inspire un certain effroi. Il se demande où va l'homme, dans quelle direction avance notre civilisation industrielle. Une suppression absolue de toute initiative personnelle est-elle en accord avec la particularité de l'homme et de ses "penchants ${ }^{11}$ » séculaires ? Ces questionnements ne concernent en aucune façon les libertés politiques et les différences idéologiques. L'écrivain prend soin de clarifier sa position et de se concentrer uniquement sur l'esprit: "est-il possible pour l'homme en tant qu'esprit de vivre éternellement privé de son individualisme même si on lui accorde en toute suffisance tous les biens et tous les conforts matériels?» En 1933 déjà, il note à propos du marxisme : «l'individu est complètement absent de sa pensée ${ }^{12}$ " et il s'agit, continue-t-il, "d'une société harmonieuse, aplanie, d'un collectivisme intenable ${ }^{13}$ ». Théotokas finit par accepter que la vérité doive se situer quelque part au milieu. Comme les communistes ont dépassé toute mesure en organisant un monde en dépit de l'individu, le capitalisme de son côté a minimisé toute perspective sociale. Les deux systèmes opposés ont ignoré chacun une part de l'homme ; «l'équilibre » - note l'écrivain - « doit se trouver quelque part, sur un point que nous n'avons pas encore découvert ».

\section{Le contact avec les Balkans}

Venons-en à présent au second point qui a marqué ce voyage de Yorgos Théotokas : le contact direct avec des écrivains d'autres pays des Balkans. À l'exception d'une anthologie de prose bulgare traduite en grec par Argis Korakas qu'il avait d'ailleurs présentée dans un article publié à Sofia dans la revue Zlatorog en février $1940^{14}$, il ne semble pas avoir eu d'autres contacts directs avec le monde littéraire des Balkans, surtout après la fin de la Seconde Guerre mondiale. Le voyage en Roumanie lui offre la possibilité non seulement de connaître le monde roumain, mais aussi celui des Bulgares et des Serbes. Ces rencontres ont dû intéresser particulièrement Théotokas puisque c'est précisément avec cette expérience qu'il entame la série de quatre articles pour décrire son voyage.

21 Le dramaturge bulgare Orlin Vassiliev, homme réservé, du même âge que Théotokas, a besoin de s'expliquer et de mettre au clair les relations entre Grecs et Bulgares. Il a besoin de rassurer le Grec et il veut être rassuré à son tour sur le fait qu'il n'y a plus de problèmes, et que les deux peuples s'apprécient, s'estiment et éprouvent le besoin d'avancer ensemble. Les deux hommes expriment réciproquement le besoin de se connaître mieux. C'est pour cette raison qu'ils se retrouvent une deuxième fois; Théotokas regrette qu'ils aient recours aux services d'une interprète et qu'ils doivent échanger en français pour l'un et en russe pour l'autre. Vassiliev manifeste un vif 
intérêt pour le festival organisé à Épidaure et exprime la volonté de la Bulgarie d'y participer. Il souligne également qu'au-delà des idéologies, le système politique communiste est une nécessité historique qui a comme but d'arriver à l'humanisme tel qu'il a été réalisé par le monde classique. Théotokas, surpris par ces propos quelque peu inattendus de son collègue bulgare, lui demande la permission de les publier dans la presse grecque. Les voici : "nous nous efforçons et nous luttons pour pousser [le système] à vaincre tout ce qu'il y a de mal en lui, afin d'arriver un jour à notre idéal qui est l'humanisme grec ${ }^{15}$.»

Quant au Serbe, il s'agit du poète Vasco Popa. Agé d'une quarantaine d'années, il est plus facile d'accès, il connaît l'Europe occidentale, il parle le français, est traduit en français et certains de ses poèmes sont même traduits en grec. Théotokas en donne d'ailleurs la référence dans son article. Popa connaît déjà la Grèce. L'échange avec lui est moins formel. Il est plus spontané, et sans avoir besoin de se justifier, il parle de l'héritage byzantin commun aux deux peuples grec et serbe et il n'hésite pas lui non plus à aller jusqu'à exprimer la nécessité de se référer à la Grèce comme source de civilisation face « à l'impasse artistique de l'Europe contemporaine ${ }^{16}$ ».

Cette vision sur les valeurs du monde grec reconnue chez les intellectuels des Balkans touche profondément Théotokas et c'est probablement parce que ce regard, à travers le monde balkanique, provient de l'espace communiste que Théotokas semble reconsidérer son opinion sur ce système politique dont l'application donne des résultats avec un certain succès social dans l'Europe de l'Est.

L'écrivain grec se trouve désormais face à un monde qui cherche lui aussi l'esprit du monde classique, non celui des études philologiques, mais celui des principes philosophiques à la recherche de l'homme.

Pour Yorgos Théotokas, classicisme et esprit grec sont le résultat de l'intégration de l'essence du drame antique dans l'expression du monde moderne au-delà des exaltations romantiques, sentimentales. Il s'agit d'un travail conscient et réfléchi au-delà d'une expression spontanée où le tragique serait associé au fatalisme à l'orientale ${ }^{17}$. C'est certainement pour ces raisons que la critique lui reproche d'être trop cérébral - c'est pour cela que ses héros, trop étudiés, manquent de souplesse et sont parfaitement prévisibles sans aucune spontanéité, mais avec une maîtrise absolue de l'expression du sentiment. Mais c'est peut-être pour ces mêmes raisons que Théotokas, face à la société communiste, face à ses collègues roumains, mais aussi serbes et bulgares, se laisse surprendre.

Comme nous l'avons déjà noté, dans les années 1930 et 1940 le communisme représente pour Théotokas la menace la plus grave et, comme seule solution face à ce danger, il propose l'union d'une Europe démocratique. Autrement dit, il est question d'une rupture sans pour autant abandonner les idéaux hérités du passé ; c'est pourquoi la volonté de retournement de l'ordre établi coexiste dans sa pensée avec un esprit anticommuniste associant nationalisme et humanisme ${ }^{18}$. Mais dans les années 1950 et 1960, ce qui menace est le nihilisme qui prend ses sources dans la pensée occidentale ${ }^{19}$. C'est pourquoi les peuples des Balkans, aux yeux de Théotokas, se retournent vers le monde classique. Comme issue à cette impasse entre la « civilisation de la machine » et l'individu, il cherche à trouver le point d'équilibre ${ }^{20}$.

27 Sans nier l'importance de l'individu, le renouveau est désormais synonyme de l'effort collectif conscient vers le changement: d'une certaine façon, il se rapproche des principes du communisme. Dix ans avant son voyage en Roumanie, Théotokas n'hésite 
pas à remarquer que la littérature grecque n'est qu'une addition de cas individuels ${ }^{21}$. Malgré ses idées libérales et le soutien à l'expression personnelle, il ne peut pas nier le besoin d'un effort collectif. Pour Théotokas, l'expression collective devient le cordon ombilical qui relie l'écrivain au devenir social et intellectuel ${ }^{22}$.

Yorgos Théotokas est heureux de constater qu'au-delà de ses sources latines qui n'ont pas cessé d'être présentes, tout Roumain qui pense manifeste un intérêt pour la Grèce. L'écrivain affirme qu'il a eu des discussions " longues $^{23}$ ", et qu'il a toujours eu affaire à des gens bien évidemment parfaitement intégrés et fidèles au régime, mais prêts à échanger librement sur diverses questions et différentes idées sans préjugés idéologiques. Il insistera d'ailleurs sur le fait que durant tout le séjour, il n'y eut aucune propagande de la part des organisateurs pour promouvoir l'idéologie communiste.

Ces considérations favorables sur le monde grec, Théotokas les rencontre à plusieurs reprises au cours de son voyage dans le pays. Il remarque que les Roumains ne cessent de marquer leurs liens avec les Grecs à travers la mise en valeur des cités grecques antiques de la côte de la mer Noire ; dans les librairies, il a constaté lui-même qu'à côté bien sûr des éditions marxistes et léninistes les gens ont accès facilement à tous les grands classiques de la littérature mondiale depuis les œuvres homériques jusqu'à celles du XIX siècle. Et malgré l'absence d'ouvrages sur la théologie, l'importance du passé byzantin est " acceptée de tous ${ }^{24}$ »; même la page phanariote qui a été fortement contestée semble, selon Théotokas, désormais tournée. Yorgos Théotokas, constantinopolitain, nous a montré son attachement à l'héritage phanariote à travers son héros Léonis, dans son roman éponyme autobiographique ${ }^{25}$. N'oublions pas non plus que son père, avocat, a été le conseiller d'Elefthérios Venizélos sur la question du Patriarcat œcuménique de Constantinople (dont le siège est toujours dans le quartier du Phanar) durant les négociations à Lausanne en 1922-1923. Il est donc heureux de constater que les Phanariotes sont "d'une certaine façon réhabilités ${ }^{26}$ »; ils sont toujours vus comme des "exploiteurs » et des "féodaux ${ }^{27}$ ", mais les Roumains leur reconnaissent désormais leur part de contribution à l'éducation et à l'organisation sociale. D'ailleurs, les Roumains sont souvent fiers de dire, continue l'écrivain, qu'ils sont d'origine grecque. Même à la réunion de l'Académie, à Bucarest, lorsqu'il a été mentionné, à l'occasion des célébrations, que Ion Luca Caragiale était d'origine grecque, l'auditoire a applaudi spontanément. Théotokas ajoute que la fille de Caragiale lui a dit que son père répétait souvent : « je suis Grec. »

L'écrivain est déçu de constater que les gens associent l'État grec contemporain à l'Espagne de Franco. Tous les représentants des pays de l'Europe de l'Est sont surpris d'apprendre que Yannis Ritsos, poète communiste engagé, accompagne Yorgos Théotokas, porteur d'un passeport légal. Le Turc Nâzim Hikmet, exilé en Union soviétique, est aussi présent à Bucarest. Il déplore la situation dans son pays et demande quelle est la position du gouvernement grec face aux œuvres d'autres écrivains communistes. Théotokas prend la parole pour assurer que les traductions ne subissent aucune censure et Ritsos continue après lui en ajoutant que le seul véritable obstacle est le problème économique. Nous pouvons rappeler à ce propos que le Parti communiste grec est loin d'être reconnu, mais les camps de concentration ont été fermés en 1952, Caramanlis a déjà entamé les discussions pour le rapprochement de la Grèce avec la Communauté économique européenne, un pacte de collaboration a été signé en 1961 et le pays connaît une petite période d'effervescence avant la trahison de 1965 qui a mené à la dictature deux ans plus tard. 

nouveau classicisme qu'il prêche et qui lui permet d'une part la continuité depuis l'Antiquité et, d'autre part, la prise de contact avec l'Europe, une Europe qui est déjà dans sa phase postclassique. Désormais, son ambition de combiner, de mettre en parallèle, le monde grec et l'esprit international, la nouveauté et le classicisme, le réel et le symbole, l'histoire et l'originalité ${ }^{29}$, son ambition, donc, semble être reconnue ou du moins recherchée aussi par les intellectuels du monde communiste des Balkans. À juste titre, il dira que « la Roumanie est un pays béni ${ }^{30} »$.

\section{BIBLIOGRAPHIE}

BOIA Lucian, 2003, la Roumanie, un pays à la frontière de l'Europe, Paris : Les Belles Lettres.

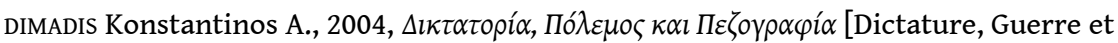

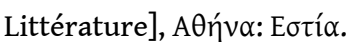

DURANDIN Catherine, 1995, Histoire des Roumains, Paris : Fayard.

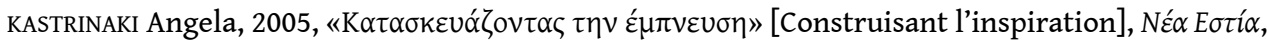
vol. $158, \mathrm{n}^{\circ} 1785$, p. 906-919.

коSTAкіотіS Georges, 2014, « Le Cheval de Moldovalachie de Georges Séféris », Cahiers balkaniques, $\mathrm{n}^{\circ} 42$, p. 131-143, mis en ligne le 27 mai 2014. URL : http://ceb.revues.org/4880 (consulté le 21 mars 2016).

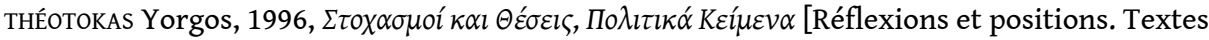

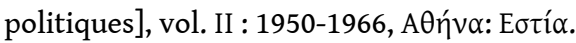

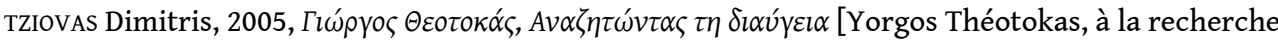

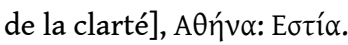




\section{NOTES}

1. Il continue à écrire des romans, des nouvelles, des pièces de théâtre; notons que

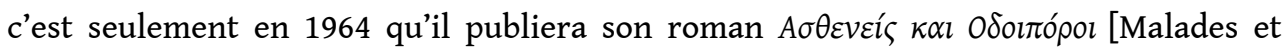
marcheurs].

2. Voir Konstantinos A. DimAdis, 2004, $\Delta \imath \kappa \tau \alpha \tau o \rho i ́ \alpha, ~ \Pi o ́ \lambda \varepsilon \mu о \varsigma \kappa \alpha l ~ \Pi \varepsilon \zeta о \gamma \rho \alpha \varphi i ́ \alpha$ [Dictature,

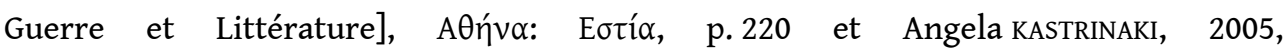

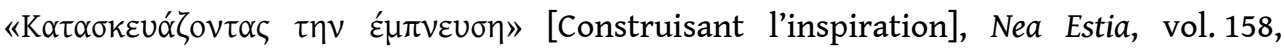
$\mathrm{n}^{\circ} 1785$, p. 916-918.

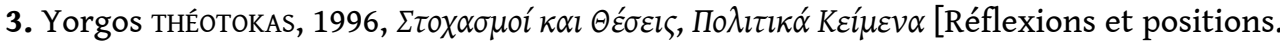

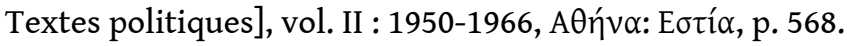

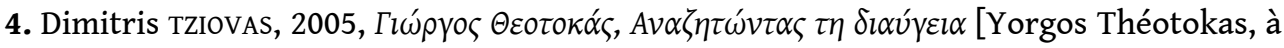

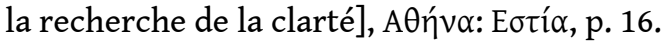

5. Yorgos THÉOTOKAS, 1996, op. cit., p. 993.

6. Ibid., p. 982.

7. Voir Catherine DURANDIN, 1995, Histoire des Roumains, Paris : Fayard, p. 387 : «On se félicite en 1961 de ce que la production industrielle de la seule capitale Bucarest est de $20 \%$ supérieure à l'ensemble de la production industrielle roumaine pour 1938 . La croissance de la population de la capitale est de 18555 habitants par an en moyenne de 1956 à 1963. L'aspect social - promotion du bien être des travailleurs - est indissociable de l'avance technique [...]. La ville nouvelle, c'est une progression de la surface bâtie et des logements ouvriers, ce sont les «blocs» qui s'alignent en des quartiers nouveaux. Les institutions et les entreprises construisent des complexes pour leurs salariés [...]. L'une des grandes réalisations des années 1950-1956 est la Casa Scînteii.» Voir également Lucian BoIA, 2003, la Roumanie, un pays à la frontière de l'Europe, Paris: Les Belles Lettres, p. 151-157.

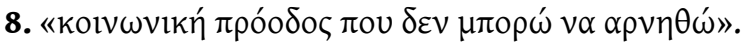

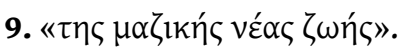

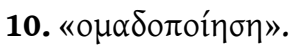

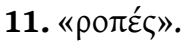

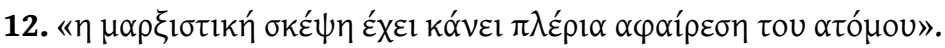

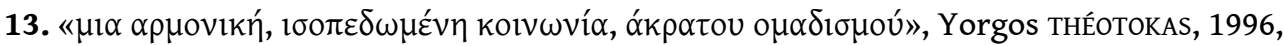
op. cit., p. 229.

14. Dimitris TZIOVAS, 2005, op. cit., p. 293.

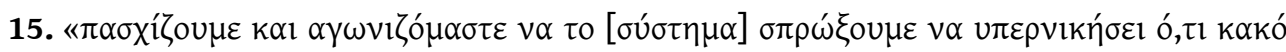

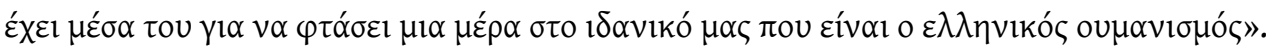

16. Yorgos тнÉотокAS, 1996, op. cit., p. 975-976.

17. Dimitris TZIOVAS, 2005, op. cit., p. 27-28.

18. Ibid., p. 33.

19. Ibid., p. 35, note 29.

20. Ibid.

21. Ibid., p. 18.

22. Ibid., p. 19. 


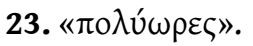

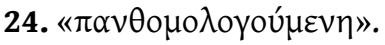

25. Yorgos тнÉотокAS, Léonis, enfant grec de Constantinople, Paris : Les Belles Lettres, 1985. Voir Georges KOSTAKIOTIS, 2012, "Georges Kostakiotis : "Images de Constantinople dans la prose néohellénique. CEuvres des $\mathrm{XIX}^{\mathrm{e}}, \mathrm{XX}^{\mathrm{e}}$ et $\mathrm{XXI}^{\mathrm{e}}$ siècles" ", Cahiers balkaniques, $\mathrm{n}^{\circ} 40$, p. 347-360, mis en ligne le 18 février 2012. URL : http://ceb.revues.org/1206 (consulté le 27 juin 2017).

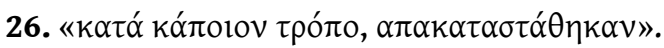

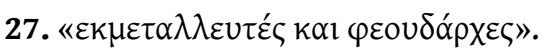

28. Georges колтакіотіS, 2014, « Le Cheval de Moldovalachie de Georges Séféris », Cahiers balkaniques, $\mathrm{n}^{\circ} 42$, p. 138, mis en ligne le 27 mai 2014. URL : https://ceb.revues.org/4880 (consulté le 21 mars 2016).

29. Dimitris TZIOVAS, 2005, op. cit., p. 31.

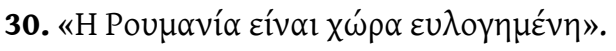

\section{RÉSUMÉS}

En 1962, à l'occasion de la commémoration en Roumanie du cinquantième anniversaire de la mort de l'écrivain-dramaturge Ion Luca Caragiale, l'écrivain Yorgos Théotokas a visité Bucarest et a séjourné en Roumanie pendant deux semaines.

Ce voyage marquera Théotokas pour deux raisons. D'une part parce que c'est la première fois qu'il a l'occasion de voyager dans un pays communiste. La Roumanie sera son premier contact avec ce monde et, pour un homme qui a combattu le communisme avec acharnement, nous pouvons dire que ce voyage restera une étape importante dans ses expériences intellectuelles. D'autre part, la Roumanie devient pour Théotokas le lieu de rencontre avec le monde balkanique; il a pu faire connaissance non seulement avec des intellectuels roumains, mais aussi avec des collègues serbes et bulgares.

Nous essaierons d'esquisser ces deux aspects afin de comprendre, d'une part l'évolution de sa pensée politique, et d'autre part le rôle qu'il accorde à la Grèce dans le monde des Balkans.

In 1962, on the occasion of the commemoration in Romania of the fiftieth anniversary of the death of writer-playwright Ion Luca Caragiale, writer George Theotokas visited Bucharest and stayed in Romania for two weeks.

This trip will mark Theotokas for two reasons. On one hand, because it is the first time he has the opportunity to travel in a communist country. Romania will be his first contact with this world and for a man who relentlessly fought communism we can say that this trip will remain a milestone for his intellectual experiences. In addition, Romania becomes for Theotokas the meeting place with the Balkan world; apart from the meetings he will have with Romanian intellectuals, the writer will have the opportunity to interact and exchange opinions with Serbian and Bulgarian colleagues.

We can therefore say that, for a man who fought with passion communism, the experience will be a milestone in spiritual pursuits. In this paper, we will try to sketch these two aspects in order 
to understand the evolution of his political thinking and also how he sees Greece in the world of the Balkans.

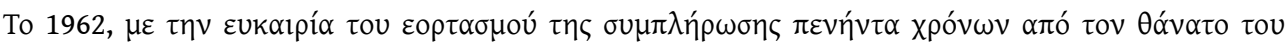

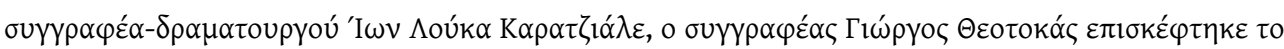

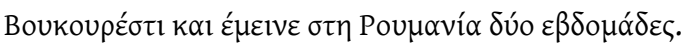

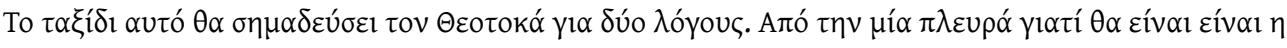

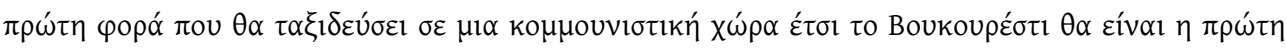

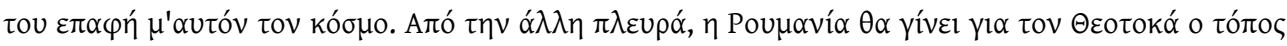

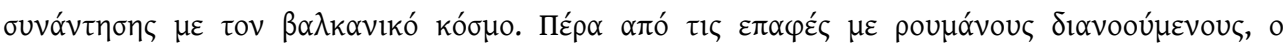

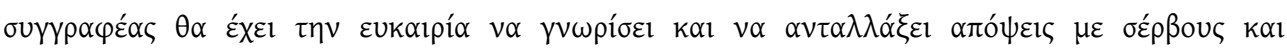

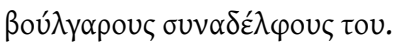

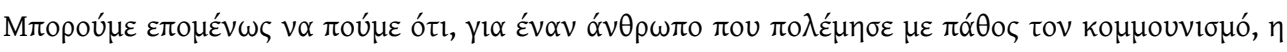

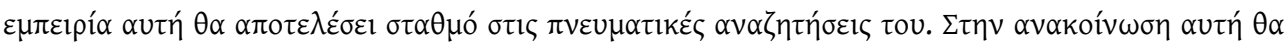

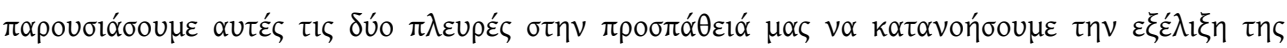

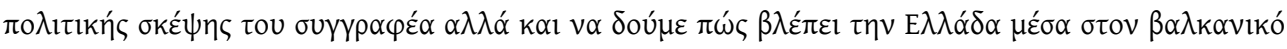

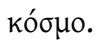

\section{INDEX}

Mots-clés : Théotokas Yorgos (1905-1966), Théotokas Yorgos (1905-1966)

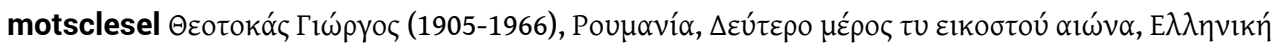

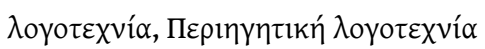

motsclestr Teotokas Yorgos (1905-1966), Romanya, Yirminci yüzyılın ikinci yarısında, Yunan edebiyatı, Seyahat edebiyatı

motsclesmk ТЕОТОКАС ЖОРЖ (1905-1966), РОМАНИЈА, ВТОРАТА ПОЛОВИНА НА ДВАЕСЕТТИОТ ВЕК, ГРЧКАТА ЛИТЕРАТУРА, ПАТУВАњЕ ЛИТЕРАТУРА

Keywords : Theotokas Yorgos (1905-1966), Romania, Second Half of the Twentieth century, Greek literature, Travelogues

Thèmes : Littérature grecque, Littérature de voyage

Index géographique : Roumanie

Index chronologique : vingtième siècle -- fin

\section{AUTEUR}

\section{GEORGES KOSTAKIOTIS}

CREE/Inalco/USPC 